\title{
Raltegravir-Induced Alopecia in an HIV+ Patient
}

Redondo $L^{1 *}$, Cristo $M^{1}$, Martìnez $Y Y^{1}$, Márquez $\mathrm{J}^{2}$, Mas $\mathbf{M}^{2}$, Ruiz S2 and Pedrol ${ }^{1}$

${ }^{1}$ Hospital of Figueres (Girona), Spain

${ }^{2}$ Hospital of Sant Pau i Santa Tecla (Tarragona), Spain

\section{Introduction}

Alopecia is defined as premature hair loss or absence of hair in one or various parts of the body. It is a condition with a high emotional impact, which can reduce the quality of life for patients and their families. It follows an unpredictable course and has shown a mixed response to established therapies.

There are two types of alopecia: scarring and none scarring. In the case of non-scarring alopecia, there is a type of hair loss that is produced by systemic diseases (including HIV) and which occurs as a side effect to the use of medication.

Alopecia related to the use of medication is due to an attack on the cells of hair follicles. Its presence affects the phases of the life cycle of hair and can differentiate between a telogen effluvium and an anagen effluvium. In the telogen effluvium phase, normal loss hair occurs due to an evolution of follicles in the anagen (growth) phase towards a premature telogen (resting) phase. These effects can be noted 2-3 months after initiating treatment. Some examples of medication that cause telogen effluvium are: vitamin A, heparin, lithium, colchicine, beta blockers, warfarin, propylthiouracil, amphetamines and carbimazole.

In the case of anagen effluvium hair loss, there is a general loss of hair during the growth phase due to the simultaneous inhibition of follicular cells undergoing division. This results in radical and extensive hair loss, beginning 7-14 days after initiating treatment. The drug which shows more evidence of anagen effluvium is daunorubicin.

Cutaneous complications caused by HIV have been noted, including a variety of opportunistic infections, psoriasis, seborrheic dermatitis and granuloma annulare, among others [1,2].

Furthermore, all groups of antiretrovirals demonstrate a level of skin toxicity to a greater or lesser extent, such as mucocutaneous dryness (zidovudine, didanosine, and lamivudine), ulcers in oral mucosa (zalcitabine), skin hyperpigmentation (zidovudine), rashes [nevirapine, efavirenz and all protease inhibitors (PI)], folliculitis, dryness, flaking and itching (nelfinavir, indinavir, ritonavir). The clinical significance of skin toxicity varies from easily controllable disorders to severe clinical manifestations that require the suspension of the antiretroviral involved.

\section{Case Presentation}

A 47 year old woman addicted to intravenous drugs. Has been $\mathrm{HIV}+$ for the past 15 years and, as a result, has received various antiretroviral treatments, which have been modified according to immunovirological failures throughout the evolution of her condition. For the past three years she has been treated with lopinavir/ritonavir (Kaletra $\odot$ ) +abacavir, as well as lamivudine (Kivexa $\odot$ ) co-formulated in such a way that they were not detectable during that same period; her CD4 lymphocytes were 420 cells/ml. During a routine visit, she described an increase in the number of bowel movements (3-5 stools per day). The decision was made to adjust her medication (following a negative microbiological analysis) and she was prescribed $400 \mathrm{mg} / 12 \mathrm{~h}$ raltegravir; lopinavir/ritonavir treatment was suspended. The patient's bowel movements normalized (one stool per day), but after a month she returned of her own accord, asking to be accessed due to hair loss in the right temporoparietal area, which she attributed to raltegravir, given that she associated the onset of alopecia with the change in medication (Figure 1). As a result and given the good immunovirological state of the patient (CV-HIV $<20$ copies $/ \mathrm{ml}$ and CD4 of 499 cells/ml), the decision was made to suspend use of raltegravir and replace it with darunavir/ ritonavir $(800 \mathrm{mg} / 100 \mathrm{mg}$ OD). After two months with this new dosage, the patient came in for a routine visit and the problem of hair loss had even resolved (Figure 2) without any loss of immunovirological control

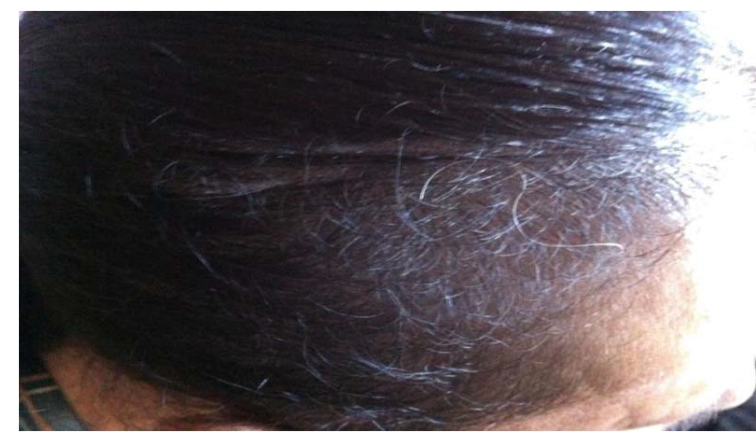

Figure 1: Alopecic area in the right fronto-parietal region one month after starting treatment with raltegravir.

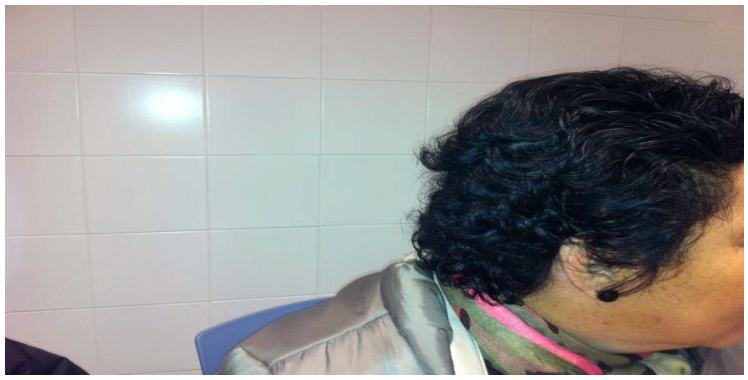

Figure 2: Regrowth of hair in the right fronto-parietal region can be observed after stopping treatment with raltegravir.

*Corresponding author: Redondo L, Doctor, Hospital de Figueres, Medicina Interna Irlanda $\mathrm{n}^{\circ} 20$, Castellar del Vallés, Barcelona 08211, Spain, Tel: +34647508372; E-mail: lauri805@hotmail.com

Received July 29, 2014; Accepted September 02, 2014; Published September 12, 2014

Citation: Redondo L, Cristo M, Martìnez YY, Márquez J, Mas M, et al. (2014) Raltegravir-Induced Alopecia in an HIV+ Patient. J AIDS Clin Res 5: 346. doi:10.4172/2155-6113.1000346

Copyright: (c) 2014 Redondo L, et al. This is an open-access article distributed under the terms of the Creative Commons Attribution License, which permits unrestricted use, distribution, and reproduction in any medium, provided the original author and source are credited. 
Citation: Redondo L, Cristo M, Martìnez YY, Márquez J, Mas M, et al. (2014) Raltegravir-Induced Alopecia in an HIV+ Patient. J AIDS Clin Res 5: 346. doi:10.4172/2155-6113.1000346

(CV-HIV RNA<20 copies/ml and CD4 lymphocytes of 514 cells/ml). One year later, the patient's condition remains stable.

\section{Discussion}

Antiretroviral drug-induced alopecia has been reported with the use of IP inhibitors and nucleoside reverse transcriptase inhibitors, particularly with lopinavir/ritonavir [3-5], which is the drug with the highest number of reported cases, and indinavir [6], both alone and in combination with ritonavir. Other antiretrovirals that have reported this side effect include ritonavir-boosted atazanavir [7], zidovudine [8] and lamivudine $[9,10]$.

Alopecia occurs primarily during the first six months after starting antiretrovirals, basically IP (average 50 days). There are some reported cases that started two to three weeks after initiation of therapy. The recovery, if it occurs, is 4-6 weeks after stopping use of the drug. A proposed pathogenic mechanism would be similar to that which occurs in retinoid toxicity. Even mechanisms involving immune reconstitution have been implicated.

Based on our knowledge and review of this subject, hair loss associated with integrase inhibitors has not been reported.

\section{References}

1. Luther J, Glesby MJ (2007) Dermatologic adverse effects of antiretroviral therapy: recognition and management. Am J Clin Dermatol 8: 221-233.

2. Xuan L, Baohua Y, Lan B (2014) Alopecia areata and vitiligo as primary presentations in a young male with human immunodeficiency virus. Indian J Dermatol 59: 209.

3. Bongiovanni M, Chiesa E, Monforte Ad, Bini T (2003) Hair loss in an HIV-1 infected woman receiving lopinavir plus ritonavir therapy as first line HAART. Dermatol Online J 9: 28.

4. Chrysos G, Mikros S, Kokkoris S, Pastelli A, Kontochristopoulos G (2007) Alopecia induced by lopinavir plus ritonavir therapy in an HIV patient. J Drugs Dermatol 6: 742-743.

5. Borrás-Blasco J, Belda A, Rosique-Robles D, Casterá E, Abad J, et al. (2007) Hair loss induced by lopinavir-ritonavir. Pharmacotherapy 27: 1215-1218.

6. Harry TC, Matthews M, Salvary I (2000) Indinavir use: associated reversible hair loss and mood disturbance. Int J STD AIDS 11: 474-476.

7. Torres HA, Barnett BJ, Arduino RC (2007) Alopecia associated with ritonavirboosted atazanavir therapy. AIDS 21: 1391-1392.

8. Chrysos G, Mikros S, Kokkoris S, Pastelli A, Kontochristopoulos G (2007) Alopecia induced by lopinavir plus ritonavir therapy in an HIV patient. J Drugs Dermatol 6: 742-743.

9. Fong IW (1994) Hair loss associated with lamivudine. Lancet 344: 1702

10. Sereti I, Sarlis NJ, Arioglu E, Turner ML, Mican JM (2001) Alopecia universalis and Graves' disease in the setting of immune restoration after highly active antiretroviral therapy. AIDS 15: 138-140. 